\title{
INVESTIGACIÓN
}

Recibido: 16/03/2021 --- Aceptado: 16/03/2021 --- Publicado: 24/05/2021

\section{LA PUPILOMETRÍA Y EL EYE TRACKING COMO HERRAMIENTAS DEL NEUROMARKETING}

\section{Pupillometry and eye tracking as neuromarketing tools}

\author{
(D) $87 R^{\circ}$ César Augusto Salazar Olarte. Universidad de León. España. \\ ddecso@unileon.es
}

Cómo citar el artículo:

Salazar Olarte, C. A. (2021). La pupilometría y el eye tracking como herramientas del neuromarketing. Vivat Academia. Revista de Comunicación, 154, 227-243. http://doi.org/10.15178/va.2021.154.e1345

http://www.vivatacademia.net/index.php/vivat/article/view/1345

\section{RESUMEN}

Estudiar el comportamiento humano en el momento de la toma de decisiones económicas es importante, debido a que las mismas están sesgadas por las emociones y los instintos. Nuestro planteamiento consiste en aportar a la demostración que los procesos cerebrales no racionales sí inciden en la toma de decisiones y que el estudio del comportamiento humano y sus decisiones debe estar involucrado con las nuevas tecnologías como el eye tracker. Esta investigación se centra exclusivamente en la utilización y pertinencia del eye tracking dentro de los procesos de atención visual selectiva, en respuesta a algunos estímulos de marketing, como es el caso de la exposición ante marcas de productos conocidas o desconocidas por el médico. Existe amplia literatura que sustenta que las fijaciones de la mirada y el diámetro de la pupila se asocian con procesos cognitivos y emocionales, demostrando que grandes dilataciones en el diámetro de la pupila se asocian a elecciones positivas, frente a pequeñas dilataciones que sugieren elecciones negativas. Se pudo establecer que el diámetro de la pupila actuó como indicador emocional ante la presentación de un video promocional de una crema para la cicatrización y tratamiento de heridas cutáneas. Adicionalmente, se determinó que el género es una variable explicativa de la diferencia de los diámetros de la pupila, y que el recorrido de la mirada y las fijaciones en determinadas áreas de interés permiten formular dos importantes conclusiones: la primera es que el conteo de fijaciones y su duración son indicadores de la atención generada en esa particular área; la segunda se relaciona a la importancia de la correcta medición del diámetro de la pupila, que permite establecer si esa fijación, producto de la atención, se debe a una respuesta emocional de aceptación o rechazo. 


\title{
Salazar Olarte, C. A. \\ La pupilometría y el eye tracking como herramientas del neuromarketing
}

PALABRAS CLAVE: Toma de decisiones - neuromarketing - comportamiento humano - eye tracking - pupilometría - atención - respuesta emocional.

\begin{abstract}
Studying human behavior when making economic decisions is important, since they are biased by emotions and instincts. Our approach consists of contributing to the demonstration that non-rational brain processes do influence decision-making and that the study of human behavior and its decisions must be involved with new technologies such as the eye tracker. This research focuses exclusively on the use and relevance of eye tracking within selective visual attention processes, in response to some marketing stimuli, such as exposure to brands of products known or unknown to the doctor. There is extensive literature that supports that gaze fixations and pupil diameter are associated with cognitive and emotional processes, showing that large dilations in the diameter of the pupil are associated with positive choices, compared to small dilations that suggest negative choices. It was possible to establish that the diameter of the pupil acted as an emotional indicator before the presentation of a promotional video of a cream for the healing and treatment of skin wounds. Additionally, it was determined that gender is an explanatory variable for the difference in pupil diameters, and that gaze travel and fixations in certain areas of interest allow two important conclusions to be drawn: the first is that the fixation count and its duration are indicators of the attention generated in that particular area; the second is related to the importance of the correct measurement of the pupil diameter, which allows us to establish whether this fixation, a product of attention, is due to an emotional response of acceptance or rejection.
\end{abstract}

KEYWORDS: Decision making - neuromarketing - human behavior - eye tracking pupillometry- attention- emotional response.

\section{INTRODUCCIÓN}

El comportamiento humano, por su complejidad, exige de estudios que van más allá de las tradicionales técnicas de observación. Estudiar el comportamiento humano en el momento de la toma de decisiones económicas es importante para ajustar la oferta de las empresas a las necesidades y deseos reales de clientes (Eraslan, Yesilada, Harper, 2014; Erel, Levy, 2016). Sin embargo, desde la perspectiva de la economía del comportamiento (Kahneman, 2003), las decisiones humanas están sesgadas emocionalmente: ¿cómo se puede determinar si la decisión tomada es emocional, racional o una mezcla de las dos, y en qué proporción? Con lo cual hay que establecer metodologías de investigación acordes con este supuesto. El propósito de este artículo es determinar si el análisis de la pupilometría es aplicable, para establecer si la decisión tomada por los individuos obedece a un proceso emocional o a un proceso racional. Para tal fin, se aplicó en el trabajo de laboratorio la tecnología eye tracker (Hübscher, Moseley, Martin, Mcauley J., 2017).

Vivat Academia. Revista de Comunicación. 2021, nº 154, 227-243 


\section{OBJETIVOS}

Demostrar que los procesos cerebrales no racionales, inciden en la toma de decisiones del ser humano.

Utilizar la pupilometría como herramienta útil de análisis del proceso emocional de toma de decisiones del ser humano.

\section{TEORÍA}

\subsection{Pupilometría}

Basados en la escuela liderada por Kahneman y Tversky, que considera que las decisiones de los seres humanos están sesgadas por las emociones y los instintos, nuestro planteamiento consiste en aportar elementos para demostrar que los procesos cerebrales no racionales inciden en la toma de decisiones. Asimismo, consideramos que tanto el estudio del comportamiento humano como sus decisiones deben estar involucrados con nuevas tecnologías como el eye tracker.

Para este propósito, existe abundante literatura que plantea, principalmente, cómo la dilatación de la pupila en los seres humanos está asociada a procesos cerebrales de aproximación/huida ante estímulos externos. Específicamente, según los autores Bayat y Pomplun (2016); Ehlers, Strauch, Georgi, Huckauf (2016); De Gee, Knapen, Donner (2014); Deltomme (2017); Kiefer, Giannopoulos, Duchowski, Raubal (2016), el diámetro de la pupila es un indicador de medida de los procesos de atención del ser humano. Adicionalmente, existe amplia literatura que sustenta que las fijaciones de la mirada y el diámetro de la pupila se asocian con procesos cognitivos y emocionales: Kahneman y Beatty (1967). De otra parte, Kiefer, Giannopoulos, Duchowski, Raubal (2016) y Bisley (2011) también plantean que el incremento en el diámetro de la pupila está implicado en los procesos de atención visual.

En tal sentido, grandes dilataciones en el diámetro de la pupila se asocian a elecciones positivas; frente a pequeñas dilataciones en el diámetro de la pupila que sugieren elecciones negativas (De Gee, Knapen y Donner, 2014).

Kinner et al. (2017) propone que la regulación de las emociones es esencial para el comportamiento adaptativo y la salud mental. Investiga los efectos de diferentes estrategias de regulación de las emociones sobre la dilatación de la pupila, las respuestas de conductancia de la piel y las respuestas emocionales subjetivas. Estos resultados indican que el diámetro de la pupila está modulado por la excitación emocional, pero que inicialmente está relacionado con el alcance del esfuerzo mental requerido para regular las respuestas emocionales automáticas.

Zenon (2016) argumenta que el diámetro de la pupila del ojo indexa la modulación del estado de excitación y responde a una gran variedad de procesos cognitivos, que incluyen esfuerzo mental, atención, sorpresa, procesos de decisión, sesgos de decisión, 
creencias de valor, incertidumbre, volatilidad, explotación/exploración, compensación o tasa de aprendizaje. En cuanto a la toma de decisiones, la dilatación de la pupila varía en contextos de incertidumbre/certeza. Cuando las decisiones se toman en ausencia de incertidumbre, como en tareas simples de asociación estímulorespuesta, la relación entre la respuesta y la ganancia de información es sencilla. En condiciones de incertidumbre, la situación es un poco más compleja. Zenon resalta que esta relación observada entre el tiempo de reacción y la dilatación pupilar se modela mejor mediante regresores que se extienden durante todo el período de tiempo de reacción de las pruebas, en lugar de pulsos breves limitados al inicio del estímulo. Estos hallazgos sugieren que el proceso a partir del cual se origina la dilatación pupilar se mantiene durante todo el proceso de decisión.

Van Slooten, Jahfari, Knapen y Theeuwes (2018) también argumentan que la pupila se dilata cuando tomamos decisiones y que estas fluctuaciones del tamaño de la pupila reflejan los cálculos de toma de decisiones durante y después de una elección. Como la mayoría de las decisiones de la vida real están guiadas por los resultados de elecciones anteriores, estos resultados muestran que las fluctuaciones del tamaño de la pupila pueden proporcionar información detallada sobre los cálculos subyacentes a las decisiones basadas en el valor y la posterior actualización de las creencias de valor.

\subsection{Eye-Tracking}

El auge de las nuevas tecnologías que permiten la interacción entre el ser humano y el ordenador, conocidas por sus siglas en inglés como HCI (Human Computer Interaction), se han vuelto accesibles para el marketing (Maibner, Musalem y Huber, 2016), ayudando a controlar desajustes o errores en los resultados de los estudios del consumidor, los cuales también pueden minimizarse sustancialmente con la aplicación de estas nuevas tecnologías.

Dentro del gran auge de las nuevas tecnologías y desde inicios de los años ochenta, el proceso de eye tracking o seguimiento ocular ha venido en aumento. La aplicación de esta herramienta abarca diversos ámbitos de estudio, desde investigaciones neurológicas hasta investigaciones de usabilidad o experiencia. Esta investigación se centra exclusivamente en la utilización y pertinencia del eye tracking dentro de los procesos de atención visual selectiva (Bisley, 2011) en respuesta a algunos estímulos de marketing, como es el caso de la exposición ante marcas de productos conocidas o desconocidas por el médico.

Teniendo en cuenta lo anterior, es pertinente aclarar cómo las HCI nos ayudan a capturar datos de los movimientos oculares. En este caso, se trata de dos cosas simples, pero que normalmente se confunden. El eye tracking es el software con el que se procesan los datos obtenidos por el eye tracker, que es el hardware encargado de grabar directamente los movimientos oculares. El trabajo del eye tracker es grabar este tipo de movimientos a las velocidades y con las aceleraciones reales. 
El ojo humano es un gran sensor de detección que se desplaza a unas velocidades casi que imperceptibles (Mercer, Baddeley, Canagarajah, 2012; Pomplun, Ritter, Velichkovsky, 1996). Su campo de visión no es tan extenso, es un campo relativamente pequeño compuesto de una elipse de ciento ochenta grados (horizontal), frente a 130 grados (vertical). La precisión del campo visual humano es de menos de dos grados y se le ha denominado el área de enfoque foveal. Esta área es la que concentra o focaliza el campo visual principal, está seguida del área parafoveal que concentra entre dos y cinco grados de agudeza visual y finaliza con un área mayor a los cinco grados que se conoce como el área periférica. En tal sentido, se puede decir que cuando el ser humano desea ver o enfocar con precisión, lo hace en el área foveal y, si desea menor precisión, va trasladándose al área parafoveal y después al área periférica. Para poder focalizar nuestra visión en algo de nuestro interés, entran en acción dos tipos de movimientos oculares, los sacádicos y las fijaciones (Courtemanche et al., 2017; Salvucci, Goldberg, 2000). Las fijaciones se encargan de situar la retina en un objetivo estacionario, para procesarlo; en tanto que el movimiento sacádico es aquel cuya tarea principal es la estabilización del ojo para poder focalizar o "estabilizar" la retina. Son movimientos extremadamente veloces que se miden en milisegundos.

Eckstein et al. (2016) analizan dos medidas de seguimiento ocular que pueden usarse para estudiar el desarrollo cognitivo y la plasticidad: dilatación de la pupila y tasa de parpadeo espontáneo. El análisis de la mirada, que puede revelar el enfoque actual de la atención (Dhar, Pradhan, Gupta, Routray, 2010); Eckstein et al., 2016), así como las estrategias cognitivas y la dilatación de la pupila - modulada por el sistema locus coeruleus-norepinefrina del cerebro, que controla la excitación y la atención fisiológica - se han utilizado como una medida de la dificultad subjetiva de la tarea, el esfuerzo mental y ganancia neural. La tasa de parpadeo espontáneo se correlaciona con los niveles de dopamina en el sistema nervioso central y puede revelar procesos de aprendizaje y comportamiento dirigidos a objetivos. El seguimiento ocular ha ocupado en gran medida la investigación de imágenes cerebrales, como una forma de estudiar los mecanismos que subyacen en el comportamiento.

Para el seguimiento visual se hace necesaria la utilización de eye trackers. Como ya se dijo, el eye tracker es el hardware encargado de grabar directamente los ojos. Su trabajo es grabar los movimientos oculares a las velocidades reales y con las aceleraciones reales.

Las tasas de muestreo de seguimiento visual varían de 25 a 2000 mediciones por segundo, lo que significa que los rastreadores más rápidos alcanzan una resolución temporal inferior a milisegundos, similar al EEG (Davidson, 1988). Es aquí donde se hace relevante la utilización de aparatos (eye trackers) de alta tecnología, que realmente graben los movimientos de los ojos y no se descalibren ante los movimientos repentinos de la cabeza o movimientos involuntarios. Los datos capturados por el eye tracker se analizan con un software denominado eye tracking que, en síntesis, lo que permite es, como mínimo, agrupar y resumir los datos de las fijaciones, los movimientos sacádicos, las coordenadas y los diámetros de las pupilas y el parpadeo. 
A la luz de estos datos, se crean los trazos o caminos recorridos por los ojos (gaze maps) o las zonas en donde se fijó más la visión o mapas de calor (heat maps).

Como se evidencia en los párrafos anteriores, es pertinente utilizar la pupilometría como indicador de los procesos emocionales. Pero, para este propósito también se hace necesaria la aplicación de tecnologías eye tracker, robustas y precisas, con lo cual la medición del diámetro de la pupila se hace más fácil, dado que es una técnica no invasiva y más precisa, si se cuenta con la configuración de los eye trackers adecuados.

\section{METODOLOGÍA}

El método es un factor muy importante en los estudios de neuromarketing. Se recomienda seguir cuidadosamente los protocolos de aplicación de las herramientas destinadas en el estudio, por ejemplo, Zenón (2019) recalca la importancia en estudios de pupilometría que las condiciones de iluminación permanezcan constantes, debido a que la pupila reacciona a los cambios en la iluminación. Así, debe tenerse en cuenta esta condición para la medición en los cambios pupilares, de manera tal de poder explicar la captura de los datos como reflejo de la respuesta pupilar al procesamiento de la información.

El presente estudio es exploratorio y de carácter descriptivo, debido a la dificultad presentada para el reclutamiento de los sujetos de estudio, que en este caso fueron profesionales de la medicina. Se logró concertar 15 citas con doctores en medicina, que se desempeñan en áreas en donde tienen contacto permanente con pacientes. De los 15 doctores a estudiar, ocho fueron hombres y siete, mujeres. Siete con edad inferior a los 29 años, seis con edad entre los 46 y 55 años, uno con edad entre 29 y 39 años y, por último, uno con edad superior a 55 años.

La prueba de pupilometría se realizó en un espacio individual, cerrado, de cuatro metros cuadrados de superficie, con luz tenue y constante a través de dos lámparas eco-halógenas. Adicionalmente, las paredes del espacio utilizado eran de color blanco opaco, que evita brillos y resplandores. Se utilizó un monitor de 17' y el eye tracker de la empresa EyeTech $(60 \mathrm{~Hz})$. El equipo se ubicó a 70 centímetros de distancia de cada uno de los participantes. Cada participante obtuvo una calibración de ambos ojos superior al 80\%. La resolución del monitor utilizada fue de 1366 x 768 pixeles. En este monitor se proyectó un video de un minuto y seis segundos de duración, en donde aparecía la presentación de una crema tópica para el tratamiento de quemaduras y úlceras en la piel. El software utilizado para el análisis de los datos obtenidos con el eye tracker fue el eye tracking Mangold Vision 4.0 (https://www.mangoldinternational.com). A cada uno de los participantes se le informó, antes de la prueba, en qué consistía la misma y se les dio una breve explicación del eye tracker, que es una tecnología no invasiva sin ningún riesgo para la salud visual del individuo.

Para el análisis de los datos obtenidos por el eye tracker se utilizó el método de agregación por fijaciones. Este método crea grupos alrededor de puntos de fijación. Para esto, se deben encontrar al menos 3 coordenadas que deben registrarse dentro de 
la distancia definida entre sí (tamaño del cluster) y tener, al menos, una fijación mínima en el momento en que se encuentran estos tres puntos. Todos los puntos dentro de esta área se agregan a un solo grupo. Esos resultados pueden leerse como puntos de fijación de la vida real y todos los valores fuera de esas áreas serán ignorados. Con este método, finalmente se obtuvo 1032 registros agregados.

A cada participante se le indicó que aparecería un video de un minuto y seis segundos de duración. Al finalizar el mismo aparecía automáticamente en la pantalla un cuestionario, con preguntas referentes al contenido del video. La captura de los datos del cuestionario se realizó a través de la aplicación CAWI (Computer Assisted Web Interviewing) de la empresa Tesi (https://www.tesigandia.com).

\section{DISCUSIÓN}

En la Figura 1 se observan las AOIs o áreas de interés predefinidas para el análisis de los datos. Son cinco áreas de interés determinadas a priori:

1. Las ubicadas en el centro de la pantalla, que van del atributo uno al atributo tres. En el área del atributo número uno aparecía la marca del producto y la siguiente frase: "Bioingeniería aplicada a la cicatrización de heridas".

2. Para el atributo número dos, también en el centro de la pantalla, aparecían constantemente imágenes de células, cadenas de células, y similares.

3. Para el atributo número tres, aparecían los siguientes textos: "Mayor tejido de granulación, apariencia, color y elasticidad similar a la normal" y "Heridas, Quemaduras, Úlceras Cutáneas".

4. La cuarta área de interés se ubicó en el extremo superior izquierdo de la pantalla, en donde continuamente aparecía la marca del producto.

5. Finalmente, el área de interés número cinco estaba ubicada en el extremo inferior derecho de la pantalla, lugar en el que siempre aparecía el logo del laboratorio farmacéutico fabricante del producto a evaluar.

El producto evaluado fue una crema tópica para la curación de heridas en la piel por quemaduras. Para este artículo no se presentan ni la marca ni el nombre del laboratorio, por razones de privacidad de estos datos.

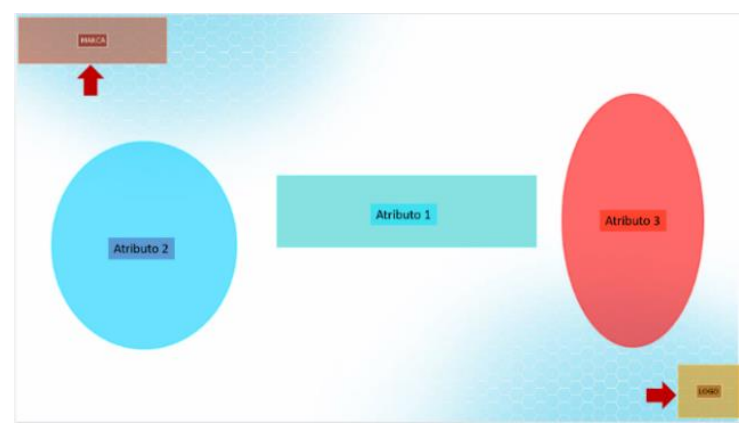

Figura 1. Áreas de interés.

Fuente: Elaboración propia. 
En la Figura 2 se presentan los cuatro mapas de calor o heat maps, como resultado de las pruebas de eye tracking aplicadas a los hombres participantes del estudio. Estos mapas de calor reflejan los momentos puntuales en donde se generó el mayor foco de atención en el video. En los mapas número uno y dos se observa que el mayor conteo de las fijaciones de la mirada se presentó para el atributo número uno y en menor intensidad para el atributo número tres. En estos momentos específicos del video no aparecían frases de refuerzo hacia el producto. En el mapa número tres continúa la tendencia de fijación hacia el atributo uno, y, para el atributo tres, ya se fija la atención en el texto de explicación o argumentación acerca de las bondades de cicatrización del producto. En el mapa número cuatro la atención se focaliza fuertemente en el efecto curativo de la crema, es decir en el atributo número uno.

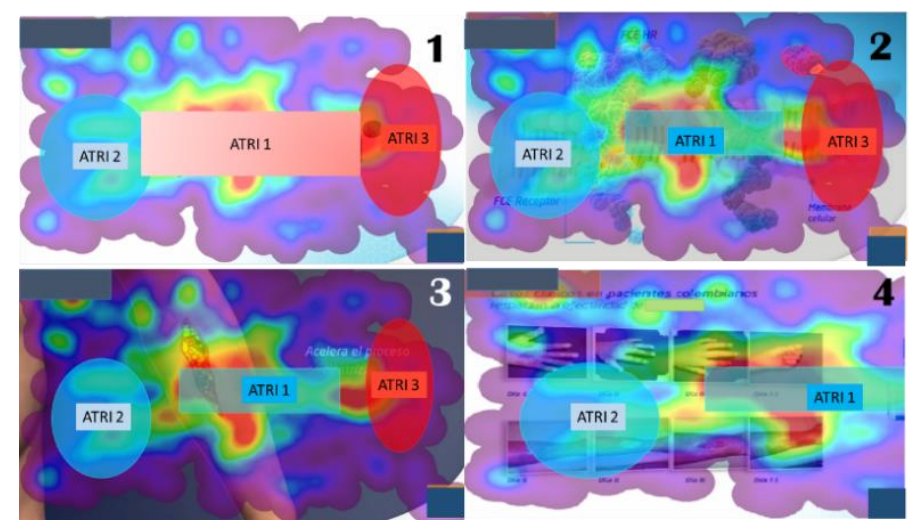

Figura 2. Fijaciones obtenidas para los hombres. Fuente: Elaboración propia.

En la Figura 3 se presentan los cuatro mapas de calor o heat maps correspondientes a las mujeres participantes del estudio. Estos mapas reflejan los momentos puntuales en donde se generó el mayor foco de atención durante el video. Los mapas de calor o heat maps resultantes son similares al comportamiento de la mirada en el grupo de los hombres, con similar tendencia hacia un marcado aumento de la atención en la demostración de la eficacia de la crema cicatrizante, reflejada en el atributo número uno del mapa número cuatro. 


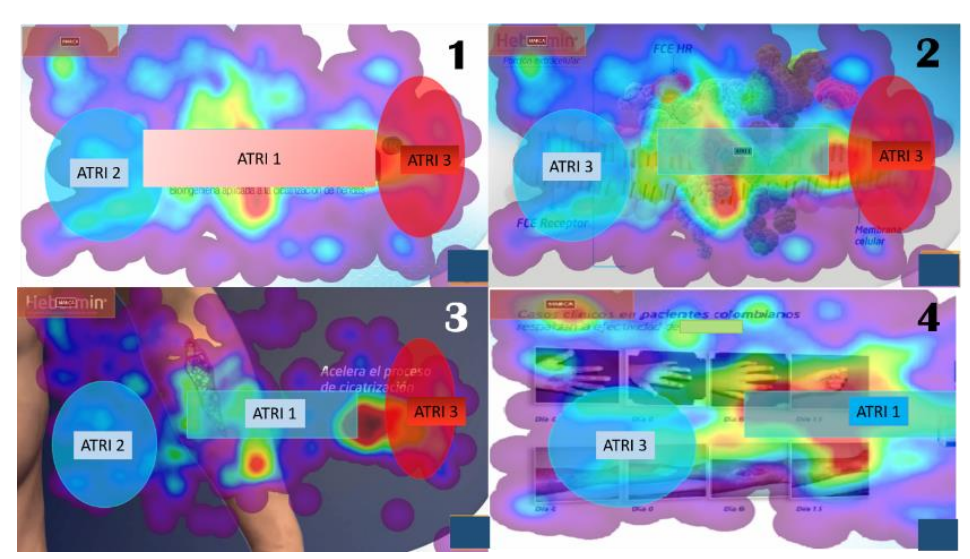

Figura 3. Fijaciones obtenidas para las mujeres.

Fuente: elaboración propia.

Ahora bien, observando en la Figura 4 la distribución de frecuencias absolutas por género, de cada una de la AOIs provenientes de los mapas de calor o heat maps de las Figuras 1 y 2, el atributo número uno obtuvo el mayor número de fijaciones agregadas, seguido de los atributos tres y dos. Las áreas de la marca y del logo, presentan bajos conteos de fijaciones agregadas y el logo del laboratorio farmacéutico no atrajo la atención de los participantes del estudio.

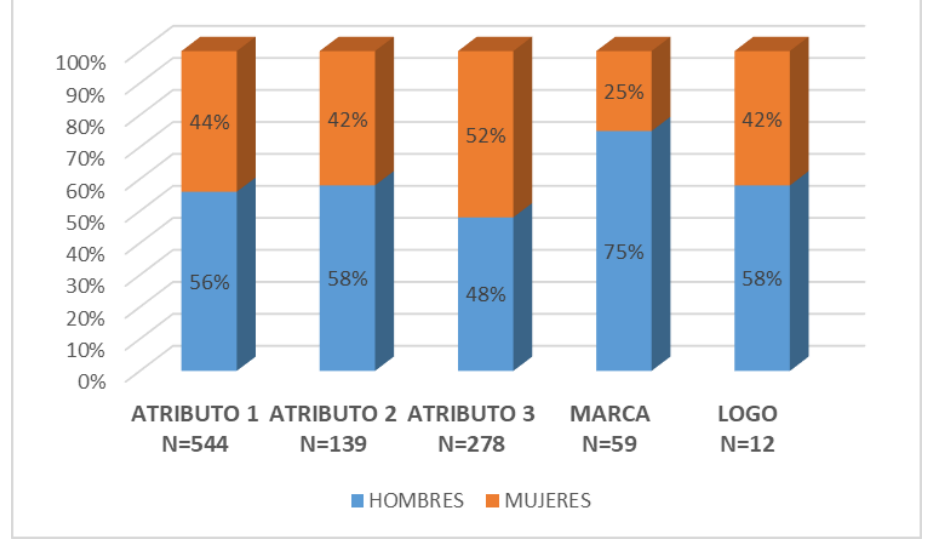

Figura 4. Frecuencias absolutas por género.

Fuente: elaboración propia.

Descriptivamente, se puede apreciar alguna incidencia de la variable género en cada una de las áreas de interés objeto del estudio. En tal sentido, se procedió con el análisis ANOVA para esclarecer si tanto la variable género como la variable área de interés incidían en la variación del diámetro de la pupila de los participantes.

Con el propósito de complementar este análisis, la Figura 5 aporta la distribución agregada de las fijaciones en relación al diámetro de la pupila observado en hombres y mujeres. El diámetro de la pupila para hombres y mujeres oscila aproximadamente entre los tres y siete milímetros. La distribución de los datos, tanto para hombres como para mujeres, no se ajusta a la curva normal. Un promedio de diámetro de la pupila menor en los hombres que en las mujeres sugiere que el proceso de fijación y atención 
fue mayor en las mujeres que en los hombres, debido a que el diámetro de la pupila en las mujeres es mayor al de los hombres.

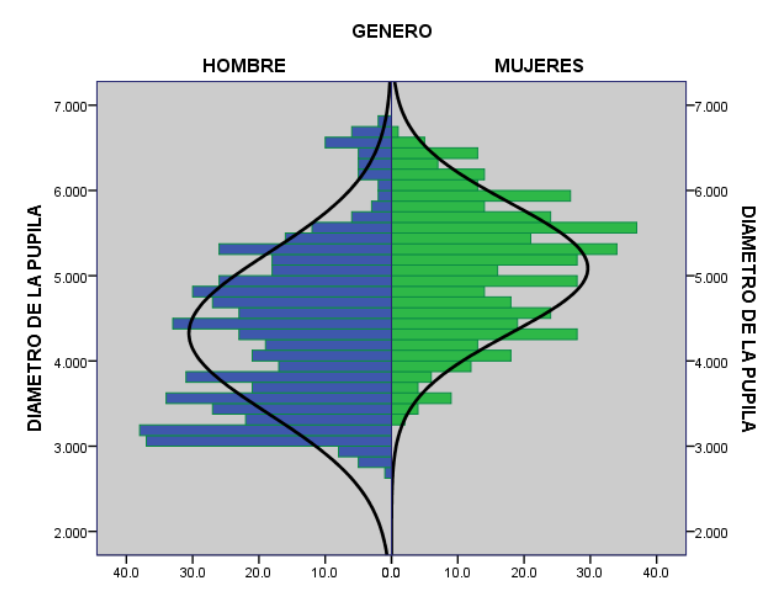

Figura 5. Distribución de la variable "diámetro de la pupila".

Fuente: Elaboración propia.

Dando continuidad al análisis de la incidencia de la variable género y particularmente la categoría mujeres, es pertinente - previo a cualquier análisis confirmatorio - aplicar la prueba de normalidad para el diámetro de la pupila a través del contraste Shapiro Wilks. Este contraste arrojó una significancia de 0.000 con 1032 grados de libertad y su estadístico de 0.982. Esto comprobó lo expuesto en el párrafo anterior, sobre la ausencia de normalidad en los datos de la variable diámetro de pupila.

Habiendo determinado la ausencia de normalidad, se aplicó el análisis de la varianza (ANOVA), con el objetivo de determinar si existían diferencias significativas en dos grupos de variables independientes. Estos grupos fueron la variable género y la variable áreas de interés. Para las variables a contrastar, se eligió el tiempo de duración (fijación) y el diámetro de la pupila. Antes de proceder con el análisis de la varianza se solicitó la prueba Levene de igualdad de varianzas para las dos variables a contrastar. En relación a la variable independiente género y la variable tiempo de duración, el estadístico arrojado fue 14.565 con significación 0.000 . A su vez, para la variable dilatación de la pupila, el estadístico de Levene fue 23.046 y significación 0.000. Esto permitió establecer para ambos casos la no igualdad de las varianzas para cada una de las variables efecto. Ahora bien, para la variable independiente áreas de interés, se repitió el mismo contraste, arrojando como estadístico de Levene 4.716 y significación de 0.001. Para el diámetro de la pupila el estadístico fue $2.571 \mathrm{y}$ significación de 0.037. Igualmente, se puede aseverar que los datos provenientes de las variables contrastadas no presentan igualdad de varianzas. Al conocer los resultados de las pruebas para el supuesto de la igualdad de las varianzas, se elige como estadístico post hoc de contraste el Games-Howell que permite contrastar los resultados de los estadísticos bajo el supuesto de diferencia en las varianzas. 
Tabla 1. Análisis de la varianza ANOVA.

\begin{tabular}{|c|c|c|c|c|}
\hline \multicolumn{2}{|c|}{} & F & Sig & Partial eta squared \\
\hline \multirow{2}{*}{ Género } & Tiempo de Duración & 147.222 & 0.000 & 0.014 \\
\cline { 2 - 5 } & Diamétro Pupila & 196.111 & 0.000 & 0.160 \\
\hline \multirow{2}{*}{ AOIs } & Tiempo de Duración & 1.759 & 0.135 & 0.007 \\
\cline { 2 - 5 } & Diamétro Pupila & 5.829 & 0.000 & 0.022 \\
\hline
\end{tabular}

Fuente: Elaboración propia.

En la Tabla 1 se presenta el resultado de las pruebas ANOVA para las variables independientes género y AOIs. La variable género incide en las dos variables contrastadas (tiempo de duración de la fijación y diámetro de la pupila), con niveles $\mathrm{p}<0.05$, sin embargo, en cuanto a la variable independiente AOIs, únicamente incide en las diferencias de las varianzas de la variable diámetro de la pupila. Adicionalmente, la prueba Games-Howell (columna Partial eta squared de la Tabla 1) de no igualdad de varianzas, al ser muy baja en la puntuación, solo nos permite aclarar que la variable independiente género incide con mayor fuerza que la variable AOIs en las dos variables independientes.

Habiéndose determinado que la variable género incide en el comportamiento del diámetro de la pupila y en las áreas de interés (AOIs), y como complemento descriptivo al análisis ANOVA, se presenta el análisis de correspondencias con el propósito de identificar las asociaciones más cercanas entre las categorías de la variable AOIs y las categorías de la variable diámetro de la pupila.

Dado que la variable diámetro de la pupila posee las características de una variable continua, se procedió a transformarla en una variable ordinal, convirtiendo esos valores a valores $Z$ con media 0 y desviación 1 . Los valores superiores a una desviación se categorizaron como "pupila dilatada", a los valores comprendidos entre 0 y 1 se les asignó la categoría "pupila normal" y a los valores inferiores a 0 se les consideró en la categoría "pupila en contracción". A través de este método se creó una nueva variable ordinal con tres categorías.

Para el procedimiento del análisis de correspondencias se utilizó el SPSS 22. Se asignó la variable independiente AOIs a las categorías de columna y a las categorías de fila se asignó la nueva variable, dilatación de la pupila. En la Tabla 2 se presentan los resultados del análisis de correspondencias, observándose que la dimensión número uno representa el $95.5 \%$ de la variación total y la dimensión número dos el $4.5 \%$. 
Tabla 2. Dimensiones del análisis de correspondencias.

\begin{tabular}{|c|c|c|c|c|c|c|c|c|}
\hline & & & & & \multicolumn{2}{c|}{$\begin{array}{c}\text { Proporcion de la inercía } \\
\text { Conteo }\end{array}$} & \multicolumn{2}{c|}{$\begin{array}{c}\text { Confianza valor propio } \\
\text { Acumulad }\end{array}$} \\
\begin{tabular}{|c|c|c|c|c|c|c|c|c|} 
Dimensiación Correlación \\
\hline 1
\end{tabular} & 0.158 & 0.025 & & & 0.955 & 0.955 & 0.03 & -0.028 \\
\hline 2 & 0.034 & 0.001 & & & 1 & 1 & 0.03 & \\
\hline Total & & 0.026 & 27.05 & 0.001 & 1 & 1 & & \\
\hline
\end{tabular}

Fuente: Elaboración propia.

En la Tabla 3, podemos ver las contribuciones relativas de cada una de las categorías de las variables (columna y fila) a las dos dimensiones que representan las asociaciones o correspondencias entre estas. La dimensión uno, que recoge o representa el $95.5 \%$ de la variación total, se ve cargada en primer lugar por la categoría pupila dilatada, seguida de la categoría pupila contraída. En las categorías de la variable AOIs, los atributos tres y dos, respectivamente, contribuyen más a esta dimensión, seguidos por el atributo uno y la marca del producto. En cuanto a la dimensión número dos, que representa el $4.5 \%$ de la varianza total, las principales contribuciones corresponden a la categoría pupila normal y al logo del laboratorio farmacéutico.

Tabla 3. Contribuciones y cargas.

\begin{tabular}{|c|l|c|c|}
\hline Variable & \multicolumn{1}{|c|}{ Código } & D1 & D2 \\
\hline Dilatación & Contracción & 93.62611 & 6.37359 \\
\hline de la & Normal & 11.5468 & 88.45359 \\
\hline Pupila & Dilatada & 99.7319 & 0.26587 \\
\hline & Atributo 1 & 89.5288 & 10.4489 \\
\hline Áreas de & Atributo 2 & 96.88145 & 3.11754 \\
\hline Interés AOIs & Atributo 3 & 99.3691 & 0.63136 \\
& Marca & 91.38765 & 8.61399 \\
& Logo & 63.34464 & 36.65255 \\
\hline
\end{tabular}

Fuente: Elaboración propia.

Observando el mapa de correspondencias de la Figura 6, y teniendo en cuenta las correspondencias relativas de la dimensión uno (D1), vemos la cercanía del atributo tres y la marca del producto con la categoría pupila dilatada; en tanto el atributo dos se aleja del atributo tres, sin correspondencia con el tamaño de la pupila. Podría esto significar que la marca y los atributos presentados en el área de interés número tres generaron mayor atención que los atributos uno y dos, junto al logo del laboratorio farmacéutico. 
Figura 6. Mapa de correspondencias.

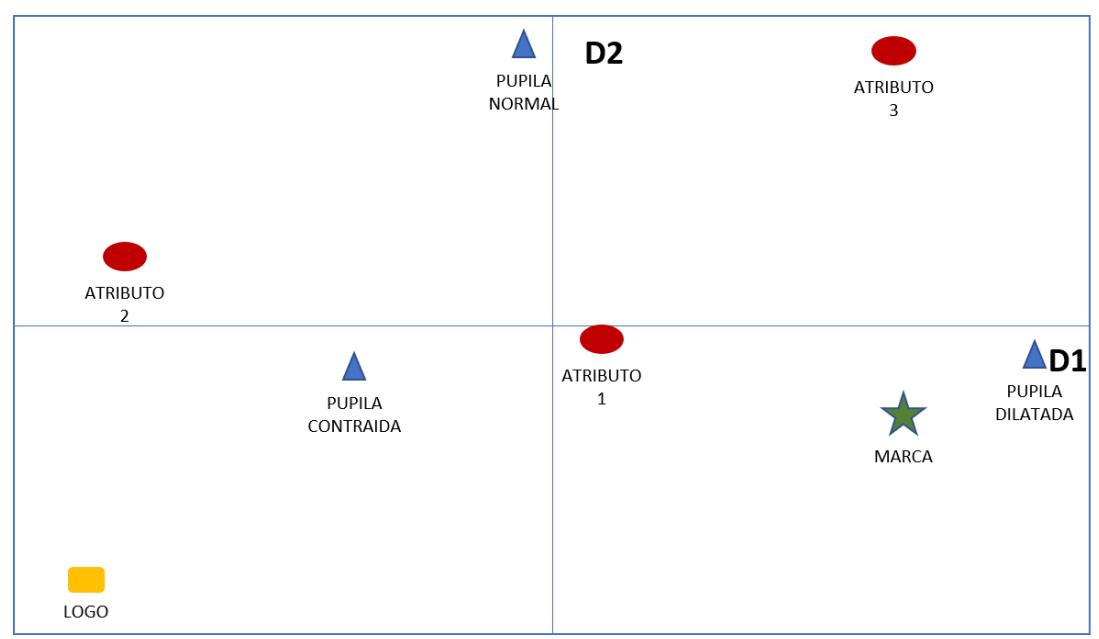

Fuente: Elaboración propia.

A continuación, se describe el análisis de las respuestas verbales a las preguntas realizadas después de ver el video. Es conveniente recordar que solicitar a una persona que responda a unas preguntas, junto a la valoración de cada uno de los ítems de respuesta, es un proceso que activa el sistema dos o el cerebro racional propuesto por Kahneman y Tversky. Aquí se resalta la importancia de cotejar o comparar descriptivamente las respuestas obtenidas en los tests de eye tracking frente a las respuestas verbales que se presentan a continuación.

La Tabla 4 recoge las respuestas a la pregunta acerca de la opinión general sobre el video, los datos representados recogen la media y el coeficiente de variación, obteniendo mayor valoración la categoría creíble, que es la que menor coeficiente de variación sustenta.

Tabla 4. Estadísticos, evaluación del vídeo.

\begin{tabular}{|c|c|c|}
\hline & Promedio & Coeficiente de variación \\
\hline IMPACTANTE & 5.267 & 24.3 \\
\hline DIFERENCIADO & 5.333 & 25.2 \\
\hline NOVEDOSO & 5.6 & 28.5 \\
\hline CREÍBLE & 6.2 & 13.9 \\
\hline
\end{tabular}


Fuente: Elaboración propia.

En la Tabla 5, se presentan los promedios y coeficientes de variación de las respuestas obtenidas a frases que recogían los atributos representados dentro de las áreas de interés. Se pudo establecer una coincidencia interesante entre las respuestas verbales y las fijaciones con sus respectivos diámetros de la pupila. El mayor promedio lo recoge uno de los atributos ubicados en el área de interés número uno, seguido del atributo número uno: "Acelera el proceso de cicatrización”.

Tabla 5. Estadísticos de los atributos.

\begin{tabular}{|l|c|c|}
\hline Frase & Media & $\begin{array}{l}\text { Coeficiente de } \\
\text { variación }\end{array}$ \\
\hline Es eficaz en el manejo de heridas y úlceras cutáneas. & 5.993 & 23.4 \\
\hline $\begin{array}{l}\text { Posee actividad bacteriostática y bactericida, protegiendo la } \\
\text { zona afectada de una posible infección. }\end{array}$ & 6.8 & 6.1 \\
\hline Reduce el tiempo de evolución de la herida. & 5.867 & 30.8 \\
\hline Acelera el proceso de cicatrización. & 6.257 & 19.5 \\
\hline $\begin{array}{l}\text { Proporciona un mayor tejido de granulación dejando una } \\
\text { piel de apariencia, color y elasticidad similares a la normal. }\end{array}$ & 6.267 & 22.1 \\
\hline
\end{tabular}

Fuente: Elaboración propia.

Finalmente, en la Tabla 6, los médicos decidían si prescribirían el producto o no. El $60 \%$ manifestó conocer el producto, pero no lo había prescrito y dijo que empezaría a prescribirlo.

Tabla 6. Frecuencias de la variable prescripción.

\begin{tabular}{|l|c|c|}
\hline & Frecuencias & Porcentajes \\
\hline Total & 15 & $100 \%$ \\
\hline Definitivamente no lo voy a prescribir a mis pacientes. & 0 & 0 \\
\hline Probablemente voy a prescribirlo a alguno de mis pacientes. & 2 & 13.3 \\
\hline $\begin{array}{l}\text { Aunque nunca lo he prescrito, voy a empezar a prescribirlo a alguno } \\
\text { de mis pacientes. }\end{array}$ & 9 & 60 \\
\hline Lo seguiré prescribiendo de igual manera a mis pacientes. & 0 & 0 \\
\hline Aumentaré la prescripción de este producto con más pacientes. & 4 & 26.7 \\
\hline
\end{tabular}

Fuente: Elaboración propia.

\section{CONCLUSIÓN}

A través del trabajo presentado en este artículo, cuyo propósito principal era determinar si el análisis de la pupilometría (medición del diámetro de la pupila) es relevante para establecer si la decisión tomada por los individuos obedece a un proceso emocional o racional, queda claro que dicho análisis es pertinente para el análisis de la toma de decisiones. A lo largo de la presente investigación se expusieron las razones por las cuales diferentes autores han utilizado la medición del diámetro de la pupila (pupilometría) en diversos ámbitos de la investigación médica y psicológica, y su importancia en el establecimiento de las reacciones emocionales sin la mediación de 
respuestas verbales. En la presente investigación se combinó, metodológicamente, la tecnología del eye tracking con la tecnología CAWI (Computer Assisted Web Interviewing), que fue la encargada de capturar las respuestas verbales de los participantes en el estudio. Se pudo establecer que el diámetro de la pupila actuó como indicador emocional ante la presentación de un video promocional de una crema para la cicatrización y tratamiento de heridas cutáneas. Adicionalmente, se determinó que el género es una variable explicativa de la diferencia de los diámetros de la pupila y que el recorrido de la mirada y las fijaciones en determinadas áreas de interés, permiten descubrir dos importantes conclusiones: la primera es la referente a que el conteo de fijaciones, junto a su duración, son indicadores de atención generada en esa particular área; la segunda se relaciona a la importancia de la correcta medición del diámetro de la pupila, lo cual permite establecer si esa fijación que produjo la atención se debe a una respuesta emocional de aceptación o rechazo. Con esto también se pudo contrastar en este estudio que las respuestas verbales de los médicos evaluados coincidían con los hallazgos de la pupilometría. En este punto, es de especial interés que en futuras investigaciones se puedan combinar las herramientas HCI (Human Computer Interface) con los instrumentos tradicionales tipo encuesta. Esta combinación permite enriquecer los hallazgos de los estudios con un amplio panorama de aplicación. Lo importante es la creatividad en los diseños metodológicos para la investigación del consumidor, en contraste con la aplicación de una sola técnica de recolección de los datos. Con los avances en la velocidad de procesamiento y en la capacidad de almacenamiento de los ordenadores, es muy alcanzable dicha creatividad e innovación.

\section{REFERENCIAS}

Bayat, A. \& Pomplun, M. (2016). The Influence of Text Difficulty Level and Topic on Eye-Movement Behavior and Pupil Size during Reading. 2nd International Conference of Signal Processing and Intelligent Systems (ICSPIS) https://doi.org/10.1109/ICSPIS.2016.7869898

Bisley, J. W. (2011). The Neural Basis of Visual Attention. The Journal of Physiology, 589(1), 49-57. https://doi.org/10.1113/jphysiol.2010.192666

Courtemanche, F., Léger P.-M., Dufresne, A., Fredette, M., Labonté-LeMoyne, E., Sénécal, S. (2017). Physiological heatmaps: a tool for visualizing users' emotional reactions. Multimed Tools Appl. https://doi.org/10.1007/s11042-017-5091-1

Davidson, R. J. (1988). EEG measures of cerebral asymmetry: conceptual and methodological issues. International Journal of Neuroscience, 39(1-2), 71-89. https:// doi.org/10.3109/00207458808985694

De Gee, J. W., Knapen, T., Donner, T. H. (2014). Decision-related pupil dilation reflects upcoming choice and individual bias. Proceedings of the National Academy of Sciences, 111. https://doi.org/10.1073/pnas.1317557111 
Deltomme, B. (2017). Acta Psychologica. https://doi.org/10.1016/j.actpsy.2017.08.010

Dhar, S., Pradhan, T., Gupta, S., Routray, A. (2010). Implementation of Real Time Visual Attention Monitoring Algorithm of Human Drivers on an Embedded Platform. Proceedings of the 2010 IEEE Students Technology Symposium 3-4, IIT Kharagpur.

Eckstein, M., Guerra-Carrillo, M., Singley, M., Bunge, A., Silvia, A. (2016). Beyond eye gaze: What else can eyetracking reveal about cognition and cognitive development. Developmental Cognitive Neuroscience. https://doi.org/10.1016/j.dcn.2016.11.001

Ehlers, J., Strauch, C., Georgi, J., Huckauf, A. (2016). Pupil Size Changes as an Active Information Channel for Biofeedback Applications. Applied Psychophysiology and Biofeedback 41(3), 331-339. https:/ / doi.org/10.1007/s10484-016-9335-z

Eraslan, S., Yesilada, Y., Harper, S. (2014). Identifying Patterns in Eyetracking Scanpaths in Terms of Visual Elements of Web Pages, S. Casteleyn, G. Rossi, and M. Winckler (Eds.). Springer International Publishing Switzerland: ICWE 2014, LNCS 8541, 163-180.

Erel, H., Levy, D. (2016). Orienting of visual attention in aging. Neuroscience and Biobehavioral Reviews, 60, 1-24. https://doi.org/ 10.1016/j.neubiorev.2016.08.010

Hübscher, M., Moseley, L., Martín, B., Mcauley, J. (2017). The reliability of eyetracking to assess attentional bias to threatening words in healthy individuals. Behavior Research Methods. https://doi.org/10.3758/s13428-017-0946-y

Hahnemann, D., \& Beatty, J. (1967). Pupillary responses in a pitch-discrimination task. Perception E Psychophysics, 2(3), 101-105. https://doi.org/10.3758/BF03210302

Kahneman, D. (2003). Maps of Bounded Rationality:Psychology for Behavioral Economics. The American Economic Review, 93(5), 1449-1475.

Kiefer, P., Giannopoulos, I., Duchowski, A. T., Raubal, M. (2016). Measuring Cognitive Load for Map Tasks through Pupil Diameter. Proceedings of the Ninth International Conference on Geographic Information Science (GIScience 2016). Springer International Publishing.

Kinner, V., Kuchinke, L., Dierolf, A., Merz, C., Otto, T., Wolf, O. (2017). What Our Eyes Tell Us About Feelings: Tracking Pupillary Responses During Emotion Regulation Processes. Psychophysiology, 54(4), 508-518. https://doi.org/10.1111/psyp.12816

Meibner, M., Musalem, A., Huber, J. (2016). Eye Tracking Reveals Processes That Enable Conjoint Choices to Become Increasingly Efficient with Practice. Journal of Marketing Research, Vol LIII, 1-17. https://doi.org/10.1509/jmr.13.0467 
Mercer Moss, F. J., Baddeley, R., Canagarajah, N. (2012). Eye Movements to Natural Images as a Function of Sex and Personality. Perception $\mathcal{E}$ Psychophysics, 2, 101-105. https://doi.org/10.1371/journal.pone.0047870

Pomplun, M., Ritter, H., Velichkovsky, B. (1996). Disambiguating complex visual information: towards communication of personal views of a scene. Perception 25(8), 931-948.

Salvucci, D. D., Goldberg, J. H. (2000). Identifying fixations and saccades in eyetracking protocols. Paper presented at the proceedings of the 2000 Symposium on eye tracking research $\mathcal{E}$ applications, Palm Beach Gardens, Florida, USA.

Van Slooten, J., Jahfari, S., Knapen, T., Theeuwes, J. (2018). How Pupil Responses Track Value-Based Decision-Making During and After Reinforcement Learning. https://doi.org/10.1371/journal.pcbi.1006632

Yuan, L., Xu, T., Yu, C. (2017). Seeing Is Not Enough for Sustained Visual Attention. Conference Paper, Department of Psychological and Brain Sciences, and Cognitive Science Program, Indiana University Bloomington, IN, 47405 USA.

Zenon, A. (2019). Eye Pupil Signals Information Gain. Proc Biol Sci, 286(1911). https://doi.org/10.1098/rspb.2019.1593

\section{AUTOR:}

\section{César Augusto Salazar Olarte}

Doctor en Nuevas Tendencias en Dirección de Empresas por la universidad de León, España. Autor del libro: Investigación Comercial y de mercados. Autor del libro: Las Nuevas habilidades comerciales del siglo XXI,2: EL cerebro detrás de las ventas. Autor de varios artículos de neuromarketing. Más de 20 años de experiencia en la investigación social y de mercados. Especialista en investigación del comportamiento humano y neurociencia aplicada al entendimiento del consumidor. Profesor y conferencista. Actualmente pertenece al grupo de investigación Mangold International de Arnstorf Alemania.

Research Gate: https://www.researchgate.net/profile/Cesar-Salazar-Olarte

ORCID: https://orcid.org/0000-0001-8636-0549

Google Scholar: $\underline{\text { https://scholar.google.com/citations?hl=es\&user=cJA19TsAAAAI }}$ 